\title{
Non-contrast 3D radial and QISS MRA for transcatheter aortic valve replacement planning
}

\author{
Akos Varga-Szemes ${ }^{1 *}$, Paola M Cannao ${ }^{1,2}$, Giuseppe Muscogiuri ${ }^{1,3}$, Matthias Renker ${ }^{1,7}$, Carlo N De Cecco ${ }^{1,3}$, \\ Shivraman Giri ${ }^{6}$, Davide Piccini ${ }^{4,5}$, Daniel H Steinberg ${ }^{1}$, Joseph U Schoepf ${ }^{1}$ \\ From 18th Annual SCMR Scientific Sessions \\ Nice, France. 4-7 February 2015
}

\section{Background}

Because of the high prevalence of renal insufficiency in patients eligible for transcatheter aortic valve replacement (TAVR), a non-contrast evaluation of the aortic root complex along with the entire vascular access route is desirable for pre-procedural evaluation. In this pilot study we proposed to test two novel investigational, non-contrast MRA techniques to develop a protocol for TAVR planning.

\section{Methods}

The study protocol was approved by the Institutional Review Board. Non-contrast MRA was performed in 7 subjects (5 healthy volunteers and 2 patients) on a $1.5 \mathrm{~T}$ system (MAGNETOM Avanto, Siemens AG, Erlangen, Germany). A prototype 3D self-navigated whole-heart radial MRA acquisition based on a spiral phyllotaxis pattern was used to assess the cardiac anatomy and the aortic root (FOV $220 \mathrm{~mm}^{3}$, TR/TE 3.1/1.5ms, flip angle $90^{\circ}$ ). This pulse sequence employs a superior-inferior bSSFP readout at the beginning of each heartbeat to correct the displacement in the k-space based on the blood pool signal. For the evaluation of the abdominal aorta and the femoral access route, both the 3D wholeheart (FOV $400 \mathrm{~mm}^{3}$, TR/TE $3.1 / 1.5 \mathrm{~ms}$, flip angle $90^{\circ}$ ) and the prototype quiescent-interval single-shot (QISS) MRA pulse sequence were used (FOV 400x260 $\mathrm{mm}^{2}$, TR/TE $3.5 / 1.4 \mathrm{~ms}$, flip angle $90^{\circ}$, acquisition length $144 \mathrm{~mm}$, number of stations 3-4). Aortic root, abdominal aorta and femoral runoff measurements were obtained and image quality was evaluated. Aortic root parameters were compared to measurements obtained by conventional cine acquisition, and abdominal aorta and femoral measurements were correlated between the 3D and QISS MRA acquisitions.

\section{Results}

Representative thoracic and abdominal images obtained by the 3D whole heart sequence, as well as abominal MIP acquired by the QISS sequence are shown in Figure 1. The acquisition time of the thoracic 3D whole-heart scan, the abdominal 3D scan, and the abdominal QISS acquisition was $6.4 \pm 1.2,6.3 \pm 1.1$, and $3.1 \pm 0.5 \mathrm{~min}$, respectively. The minimum and maximum diameter, the perimeter, and the area of the aortic root were not different between the 3D whole-heart and the 2D bSSFP cine acquisitions (Table 1). No significant difference was found in the diameter of the abdominal aorta or the iliac and femoral arteries between the 3D and the QISS acquisitions (Table 1). The 3D whole heart acquisition provided a significantly better contrast-to-noise ratio (CNR) compared to the 2D bSSFP cine, however, CNR was not significantly different between images obtained by the 3D and QISS MRA protocols (Table 1).

\section{Conclusions}

These preliminary results suggest that the 3D wholeheart acquisition technique provides rapid, free-breathing assessment of the cardiac and aortic root anatomy without the administration of contrast medium. Although the $3 \mathrm{D}$ whole-heart acquisition also enables the assessment of the abdominal vascular status, the QISS MRA provides significantly faster evaluation of the femoral access route, which improves patient compliance.

\section{Funding}

N/A.

${ }^{1}$ Medical University of South Carolina, Charleston, SC, USA

Full list of author information is available at the end of the article 


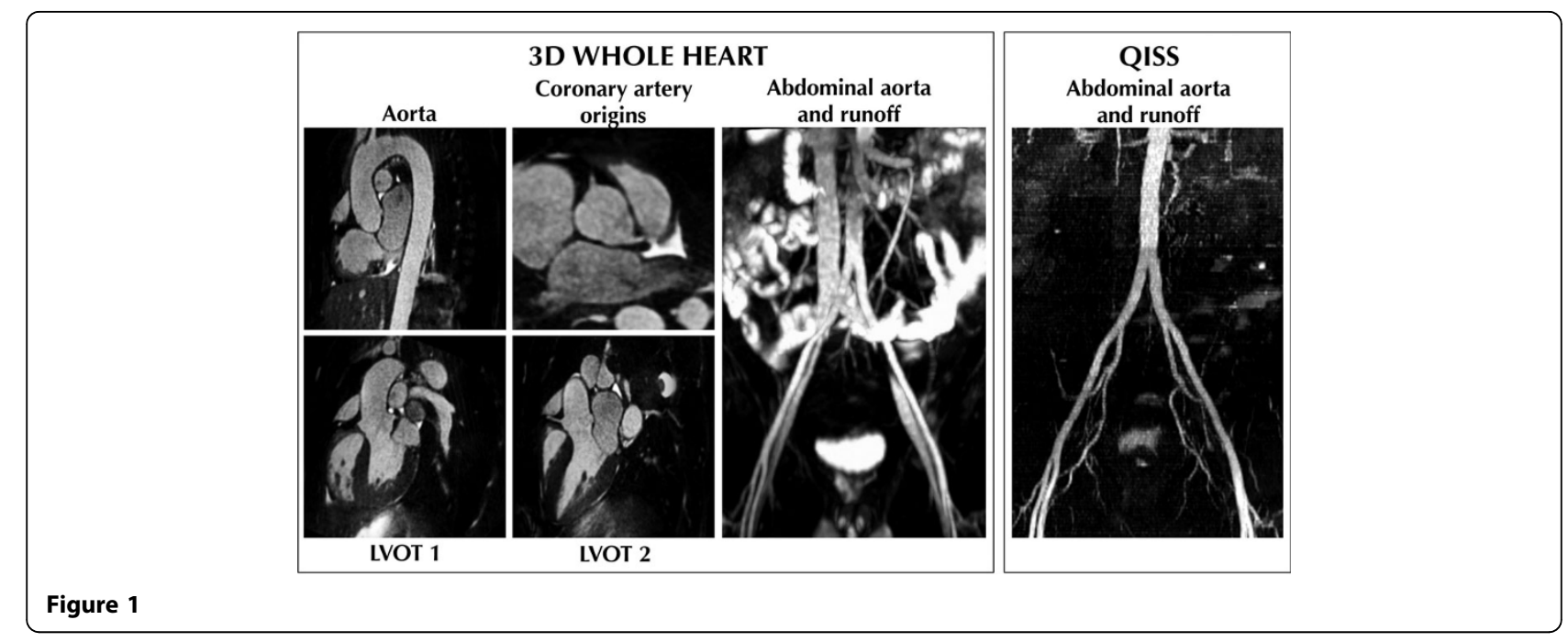

Table 1 Aortic root, abdominal and femoral vascular assessment

\begin{tabular}{|c|c|c|c|}
\hline Aortic root assessment & 3D Whole Heart & 2D-bSSFP & $P$ \\
\hline Aortic annulus diameter - min & $22.3 \pm 1.7$ & $22.6 \pm 1.5$ & NS \\
\hline Aortic annulus diameter - max & $28.2 \pm 1.4$ & $28.1 \pm 1.4$ & NS \\
\hline Aortic annulus perimeter & $89.4 \pm 6.9$ & $90.3 \pm 6.6$ & NS \\
\hline Aortic annulus area & $5.4 \pm 0.4$ & $5.6 \pm 0.3$ & NS \\
\hline CNR - valvular plane & $42.3 \pm 28.4$ & $11.9 \pm 2.7$ & $<0.05$ \\
\hline CNR - aortic root & $47.6 \pm 30.3$ & $11.4 \pm 2.9$ & $<0.05$ \\
\hline Abdominal and femoral vascular assessment & 3D Whole Heart & QISS & $P$ \\
\hline Abdominal aorta diameter & $20.8 \pm 5.5$ & $19.9 \pm 5.7$ & NS \\
\hline Left common iliac artery diameter & $11.5 \pm 0.9$ & $12.4 \pm 2.8$ & NS \\
\hline Right common iliac artery diameter & $11.8 \pm 1.9$ & $10.6 \pm 4.1$ & NS \\
\hline Left common femoral artery diameter & $10.6 \pm 0.9$ & $8.4 \pm 4.6$ & NS \\
\hline Right common femoral artery diameter & $11.2 \pm 1.1$ & $8.8 \pm 3.6$ & NS \\
\hline CNR - abdominal aorta & $12.3 \pm 4.7$ & $21.1 \pm 12.3$ & NS \\
\hline CNR - left common iliac artery & $13.2 \pm 8.3$ & $20.3 \pm 9.1$ & NS \\
\hline CNR - right common iliac artery & $18.9 \pm 11.0$ & $14.2 \pm 4.4$ & NS \\
\hline CNR - left common femoral artery & $15.2 \pm 6.9$ & $17.9 \pm 9.2$ & NS \\
\hline CNR - right common femoral artery & $18.7 \pm 11.0$ & $21.0 \pm 11.4$ & NS \\
\hline
\end{tabular}

CNR, contrast-to-noise ratio; NS, non-significant

\section{Authors' details}

${ }^{1}$ Medical University of South Carolina, Charleston, SC, USA. ${ }^{2}$ University of Milan, Milan, Italy. ${ }^{3}$ University of Rome "Sapienza", Rome, Italy. ${ }^{4}$ Siemens Healthcare IM BM IP, Lausanne, Switzerland. ${ }^{5}$ University of Lausanne, Lausanne, Switzerland. ${ }^{6}$ Siemens Medical Solutions, Chicago, IL, USA. ${ }^{7}$ University Hospital Giessen and Marburg, Giessen, Germany.

Published: 3 February 2015

\section{doi:10.1186/1532-429X-17-S1-071}

Cite this article as: Varga-Szemes et al: Non-contrast 3D radial and QISS MRA for transcatheter aortic valve replacement planning. Journal of

Cardiovascular Magnetic Resonance 2015 17(Suppl 1):O71.
Submit your next manuscript to BioMed Central and take full advantage of:

- Convenient online submission

- Thorough peer review

- No space constraints or color figure charges

- Immediate publication on acceptance

- Inclusion in PubMed, CAS, Scopus and Google Scholar

- Research which is freely available for redistribution 